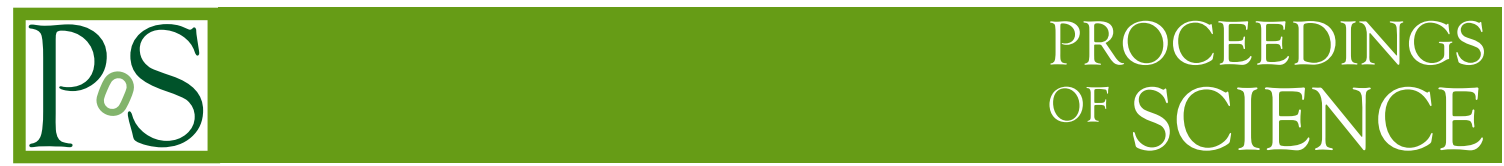

\title{
Quarkonia probes of QCD matter
}

\section{Marzia Nardi*}

Istituto Nazionale di Fisica Nucleare, Sezione di Torino

E-mail: marzia.nardi@to.infn.it

Quarkonium production and suppression is one of the most interesting observables studied and measured in relativistic heavy-ion collisions. Recent progress in theoretical calculations are presented.

International Conference on Hard and Electromagnetic Probes of High-Energy Nuclear Collisions 30 September - 5 October 2018

Aix-Les-Bains, Savoie, France

* Speaker. 


\section{Introduction}

$J / \psi$ suppression in relativistic Heavy-Ion Collisions (HIC) has been proposed as a useful probe of Quark-Gluon Plasma (QGP) formation in the laboratory [1]. It was argued that the size of the meson (i.e. the binding radius, estimated in potential models) is so small and the binding energy (i.e. the energy required to separate the two heavy quarks to form a $D-\bar{D}$ pair by creating a light quark-antiquark pair) is so strong that the interaction with normal hadrons can not break the charmonium state. But the color screening in the deconfined phase can prevent the binding of the $c-\bar{c}$ quarks and thus the $J / \psi$ formation. This simple idea is applicable to other heavy-quarks bound states: higher resonances of $c-\bar{c}$, but also of $b-\bar{b}(\Upsilon$ family) and it was at the origin of an intensive activity of many experimental Collaborations to measure the predicted effect of $J / \psi$ suppression in HIC with respect to proton-proton collision at the same energy.

It was, however, immediately realized that other phenomena are at play in such complicated events and it was not trivial to isolate the quarkonium suppression as the genuine signal of QGP formation. This fact triggered an intensive theoretical study over the past years. Moreover, it was also noticed that at RHIC and LHC energies the $c-\bar{c}$ pairs are created with such a high abundancy that, although they separate in the deconfined phase, they have a non-negligible probability to recombine in the hadronization process, giving rise, therefore, to a $J \psi$ enhancement [2]!

From a theoretical point of view, quarkonium bound states are interesting by themselves because the creation of $c-\bar{c}$ or $b-\bar{b}$ (in general $Q-\bar{Q}$ ) is calculable by Quantum Chromodynamics (QCD), due to the high scale provided by the large mass of the heavy quarks.

On the other hand, the formation of the bound states involves non-perturbative effects and proton-proton collision experiments are important to understand this part. Section 1 is devoted to a discussion of important results on this subject.

Moreover, the medium created in ultrarelativistic heavy-ion collisions can modify the way in which a newly created $Q-\bar{Q}$ pair evolves into a quarkonium bound state, and can even prevent its formation. Thus proton-nucleus experiments offer the unique opportunity to investigate this modification; this will be briefly discussed in Section 2.

Finally, Section 3 will present the results of quarkonium production and suppression/enhancement in nucleus-nucleus collisions: all phenomena that can modify the production of heavy quarks in nuclear collisions with respect to proton-proton collisions without invoking the creation of a QGP, like the ones occurring in proton-nucleus interactions, must be carefully calculated to disentangle the suppression due to the QGP formation.

\section{Quarkonium production in proton-proton collisions}

In the production process of quarkonia, both perturbative and non-perturbative aspects of QCD are involved. The production of the $Q \bar{Q}$ pair, which will subsequently form the quarkonium, can be described with high precision by perturbative-QCD, since it involves momentum transfers at least as large as the mass of the heavy quark. But the subsequent evolution of the heavy quark pair to form the bound quarkonium state is non-perturbative, since it occurs with typical momentum scales of the order of the momentum of the heavy quarks in the bound-state rest frame $m_{Q} v$ and 
of their binding energy $m_{Q} v^{2}, v$ being the typical velocity of the heavy quark or antiquark in the quarkonium rest frame $\left(v^{2}=0.3\right.$ for the charmonium and 0.1 for the bottomonium).

In nearly all the models for quarkonium production mechanisms currently used, the idea of a factorisation between the pair production and its binding is assumed. In the following, I briefly present the most popular models.

The first attempt to describe quarkonium production was done with the Colour-Singlet Model (CSM) $[3,4,5]$ which relies on the assumption that the quantum state of the pair does not evolve between its production and its hadronisation, neither in spin, nor in colour. The partonic cross section for quarkonium production involves that for the production of a heavy-quark pair with zero relative velocity $v$ in a colour-singlet state and in the same angular momentum and spin state as that of the final quarkonium.

Unfortunately, the predictions of this model were shown to dramatically disagree with experimental data of direct $J / \psi$ and $\psi(2 \mathrm{~S})$ production at $\sqrt{s}=1.8 \mathrm{TeV}$ [6], an order of magnitude larger. These discrepancies motivated new theoretical investigations on quarkonium hadroproduction, leading to the non-relativistic-QCD (NRQCD) factorisation framework [7]. In this approach, quarkonium production can also proceed via creation of colour-octet $Q \bar{Q}$ pairs, present in higherFock states, whose effects are believed to be suppressed by powers of the relative $Q \bar{Q}$ velocity, $v$.

By taking into account higher-order colour singlet contributions, i.e. next-to-leading order (NLO) corrections and approximate next-to-next-to-leading order (NNLO) contributions (known as $\mathrm{NNLO}^{*}$ ) the discrepancies of the CSM are significantly reduced [8]. A full NNLO computation is however needed to confirm this trend.

The Colour Evaporation Model (CEM) $[9,10]$ is based on the principle of quark-hadron duality; the production cross section of quarkonia is related to the one of a $Q \bar{Q}$ pair between the kinematical threshold to produce a quark pair, and that to create the lightest open heavy-flavour hadron pair. One phenomenological parameter per quarkonium state, related to a process-independent probability that the pair hadronises into this state, is introduced and it has to be extracted by fitting experimental data. This simple model is phenomenologically successful, but in its original version it suffers for discrepancies in some transverse momentum spectra and the absence of predictions for polarisation observables. Therefore an improved version of the model, the ICEM was recently proposed [11], in which the average final state momentum, $p_{\psi}$ is related to the $c \bar{c}$ pair momentum, improving the results at high $p_{T}$. Moreover, by including an explicit charmonium mass dependence, the predicted ratio of differential cross sections of two charmonium states is no longer $p_{T}$-independent. A LO calculation of quarkonium polarisation in this framework can also be developed, showing longitudinal polarisation [12].

A very recent development combines the Color Glass Condensate (CGC) saturation model of gluon distributions in the proton with NLO NRQCD matrix elements [13]. This formalism has been applied to compute $J / \psi$ production at low $p_{T}$ in proton-proton collisions at collider energies. A very good description of the total cross sections, the rapidity distributions and the low momentum $p_{T}$ distributions is obtained. Moreover, the results in this framework can be matched smoothly to NLO perturbative QCD results at high $p_{T}$, providing a unified description for quarkonium production in all phase space. 


\section{Quarkonium production in proton-nucleus collisions}

Besides the predicted colour screening preventing the $Q \bar{Q}$ pair binding, other effects, not necessarily related to the QGP formation, can lead to a suppression of quarkonium states. Some of these effects are present already in $p A$ collisions and are usually called Cold Nuclear Matter (CNM) effects.

A careful experimental and theoretical analysis is very important to separate these effects from the signature of the deconfined phase and to correctly interpret nucleus-nucleus data.

First of all, it is well known from phenomenology that the nuclear distribution functions of partons in nucleons inside nuclei differ from those measured in free nucleons. In a collinearfactorisation framework, this effects can be described in terms of nuclear-modified parton distribution functions (nPDF). Three regimes are usually identified for the nPDF to PDF ratio $R_{i}\left(x, Q^{2}\right)$, depending on the value of $x$ (the fraction of longitudinal momentum carried from the considered parton):

- a suppression $\left(R_{i}<1\right)$, commonly referred to as shadowing, at small $x<10^{-2}$;

- an enhancement $\left(R_{i}>1\right)$, known as antishadowing, at intermediate values $10^{-2}<x<10^{-1}$,

- the EMC effect, a depletion observed at large $x>10^{-1}$.

The $R_{g}\left(x, Q^{2}\right)$ parametrisations are determined by performing global fit analyses of lepton-nucleus and proton-nucleus data[14].

The modification of gluon densities in nuclei affects the yields of quarkonium production, since it implies that the nucleus-nucleus interaction is not a simple superposition of nucleonnucleon collisions. The present LHC data on quarkonium are compatible with strong shadowing in the forward rapidity region and with some antishadowing in the backward region [15].

In the forward rapidity region, parton saturation effects, described by the Color Glass Condensate (CGC) effective theory, become important because the density of gluons at small $x$ is very high. Taking into account small- $x$ evolution in the NRQCD formalism [16] and the colour-channel dependence of the hadronisation process the results for $J / \psi$ production are in good agreement with experimental data of the ALICE and LHCb Collaborations.

In a nuclear environment, the $Q \bar{Q}$ pair produced in the primary collision can interact and be destroyed by inelastic scatterings with the nucleus remnants. This was clearly observed at SPS energies, were the $J / \psi$ measured in $p A$ collisions were significantly suppressed with respect to what expected by assuming simple scaling with the number of nucleon-nucleon collisions. This effect is known as nuclear absorption.

At LHC-energies, however, the quarkonium formation time is much larger than the nucleus radius, because it is Lorentz-dilated by a factor 1000 or more, therefore the nuclear absorption is expected to be negligible. Nevertheless, other effects can be important. In particular, a coherent energy loss, depending on the projectile energy, has been proposed [17]. It can reproduce the data from fixed-target to LHC energies. In presence of such an energy loss, which arises from interferences between initial and final-state radiations, no additional break-up is needed. It is however not clear if gluon shadowing is needed when such an energy loss is at play. A possible way to disentangle shadowing from coherent energy loss is with the Drell-Yan process measurements [18]. 
Measurements of $J / \psi$ and $\psi(2 S)$ production in proton(deuteron)-nucleus collisions, at energies from SPS to LHC, show a stronger suppression for the excited state $\psi(2 s)$ than for the lower state $J / \psi$. The same occurs for the bottomonium family: the excited $\Upsilon(2 S)$ and $\Upsilon(3 S)$ states suffer more suppression than the lower $\Upsilon(1 S)$ state.

At low collision energies, such a relative suppression is naturally explained from final-state interactions with the remnants of the colliding nucleus, the already mentioned nuclear absorption. But at LHC energies the produced $Q \bar{Q}$ pair does not have the time to evolve into any physical state when it escapes the nuclear matter contained in the colliding nucleus. Consequently, one cannot invoke this mechanism to explain the relative suppression, neither can one invoke initialstate effects such as the modification of the nPDFs or the coherent energy loss, which are known to have a similar impact on the different states.

In fact, as for now, the approach which can successfully describe these results is the comover interaction model [19, 20, 21]. Such an approach accounts for final-state scatterings with comoving particles, i.e. particles (partons or hadrons) with similar rapidities that happen to travel along with the $Q \bar{Q}$ pair. These interactions can occur over times long enough for the quarkonium to be formed; differences between the different quarkonium-state suppression can be naturally explained.

\section{Quarkonium production in nucleus-nucleus collisions}

More than 30 years ago Matsui and Satz [1] proposed the suppression of quarkonium as a signature of the formation of a QGP. The original idea is that, due to the Debye-screening of the $Q \bar{Q}$ potential in the deconfined medium, different quarkonium states will melt at different temperatures, giving rise to the so-called "sequential melting".

Moreover, at high energies (RHIC, LHC), the high abundancy of charm quarks and antiquarks gives a significant probability that charmonia states can be formed at the confinement transition by recombination. In fact, some models can explain experimental data only by assuming a large fraction of $J / \psi$ 's originated in the final state regeneration. This mechanism was negligible at SPS because the number of $c \bar{c}$ pairs is very small, making very unlikely for them to meat each other at the hadronisation to form the bound state (for the same reason it is still negligible for the bottomonium at RHIC and LHC energies: the number of $b \bar{b}$ pairs are comparable with the number of $c \bar{c}$ at RHIC, but distributed in a larger volume). These effects are expected to have a different weight in different kinematical regions. Therefore to disentangle the contribution of suppression and recombination in the final charmonium yield, one has to study its whole rapidity and $p_{T}$ dependence.

The first theoretical works on $J \psi$ suppression relied on a time-independent notion of the melting process, based on purely real-model potentials, assumed to be coincident with the colour-singlet free energy, the colour-singlet internal energy or even linear combinations of both quantities.

It was subsequently found that the heavy-quark potential at first non-trivial order in resummed perturbation theory not only shows Debye screening but also has an imaginary part [22], related to the Landau damping due to the inelastic scatterings of the pair with the deconfined partons.

The dynamics of the in-medium evolution of the heavy-quark bound state can also be studied, from first-principle QCD, in lattice calculations, with complex in-medium heavy-quark potential [23] 
In a recent work [24], the derivation of equations of motion for the reduced density matrix of a heavy quarkonium in contact with a quark-gluon plasma in thermal equilibrium is shown. The effect of collisions is accounted for by an imaginary potential.

The bottomonium yields in relativistic heavy-ion collisions is estimated in recent works using a lattice QCD-inspired heavy-quark potential, with complex-values [25, 26]. The heavy quarks move in a realistic, hydrodynamically evolving medium background and the yield of quarkonia is found to be mostly sensitive to the temperature of the medium, thus strengthening the picture of bottomonium as QGP thermometer. The results are in good agreement with experimental data provided by RHIC, but the LHC data are underestimated.

\section{Conclusions}

In recent years there have been a considerable progress in the theoretical description of quarkoniun evolution in a deconfined medium, with the introduction of a static complex potential and out-of-equilibrium dynamics.

The fact that many effects can sum up to give the observed quarkonium yield means that many experiments must be performed and compared to disentangle the contributions of various origin: iniatial state (cold nuclear matter), suppression, recombination.

We have understood a lot about quarkonia in heavy-ion collisions in these years, but a lot more is still to be learned.

\section{References}

[1] T. Matsui and H. Satz, J/ $\psi$ Suppression by Quark-Gluon Plasma Formation, Phys. Lett. B 178 (1986) 416.

[2] R. L. Thews, M. Schroedter and J. Rafelski, Enhanced J/ $\psi$ production in deconfined quark matter, Phys. Rev. C 63, 054905 (2001); P. Braun-Munzinger and J. Stachel, (Non)thermal aspects of charmonium production and a new look at J / psi suppression, Phys. Lett. B 490, 196 (2000)

[3] M. B. Einhorn, S. D. Ellis, Hadronic Production of the New Resonances: Probing Gluon Distributions, Phys. Rev. D12 (1975) 2007.

[4] C.-H. Chang, Hadronic Production of J/ $\psi$ Associated With a Gluon, Nucl. Phys. B172 (1980) $425-434$.

[5] R. Baier, R. Ruckl, Hadronic Production of J/psi and Upsilon: Transverse Momentum Distributions, Phys. Lett. 102B (1981) 364-370.

[6] F. Abe, et al., $J / \psi$ and $\psi(2 S)$ production in $p \bar{p}$ collisions at $\sqrt{s}=1.8 \mathrm{TeV}$, Phys. Rev. Lett. 79 (1997) $572-577$.

[7] G. T. Bodwin, E. Braaten, G. P. Lepage, Rigorous QCD predictions for decays of P wave quarkonia, Phys. Rev. D46 (1992) R1914-R1918.

[8] P. Artoisenet, J. M. Campbell, J. P. Lansberg, F. Maltoni, F. Tramontano, ؟ Production at Fermilab Tevatron and LHC Energies, Phys. Rev. Lett. 101 (2008) 152001.

[9] H. Fritzsch, Producing Heavy Quark Flavors in Hadronic Collisions: A Test of Quantum Chromodynamics, Phys. Lett. 67B (1977) 217-221. 
[10] F. Halzen, Cvc for Gluons and Hadroproduction of Quark Flavors, Phys. Lett. 69B (1977) 105-108.

[11] Y.-Q. Ma, R. Vogt, Quarkonium Production in an Improved Color Evaporation Model, Phys. Rev. D94 (11) (2016) 114029.

[12] V. Cheung, R. Vogt, Polarization of prompt $J / \psi$ and $\Upsilon(1 S)$ production in the color evaporation model, Phys. Rev. D96 (5) (2017) 054014.

[13] Y.-Q. Ma, R. Venugopalan, Comprehensive Description of J/ÏL Production in Proton-Proton Collisions at Collider Energies, Phys. Rev. Lett. 113 (19) (2014) 192301.

[14] K. J. Eskola, P. Paakkinen, H. Paukkunen, C. A. Salgado, EPPS16: Nuclear parton distributions with LHC data, Eur. Phys. J. C77 (3) (2017) 163.

[15] J.-P. Lansberg, H.-S. Shao, Towards an automated tool to evaluate the impact of the nuclear modification of the gluon density on quarkonium, D and B meson production in proton-nucleus collisions, Eur. Phys. J. C77 (1) (2017) 1.

[16] Y.-Q. Ma, R. Venugopalan, H.-F. Zhang, J/ $\psi$ production and suppression in high energy proton-nucleus collisions, Phys. Rev. D92 (2015) 071901.

[17] F. Arleo, S. Peigne, T. Sami, Revisiting scaling properties of medium-induced gluon radiation, Phys. Rev. D83 (2011) 114036.

[18] F. Arleo, S. Peigné, Disentangling Shadowing from Coherent Energy Loss using the Drell-Yan Process, Phys. Rev. D95 (1) (2017) 011502.

[19] E. G. Ferreiro, Charmonium dissociation and recombination at LHC: Revisiting comovers, Phys. Lett. B731 (2014) 57-63.

[20] X. Du, R. Rapp, In-Medium Charmonium Production in Proton-Nucleus Collisions

[21] Y.-Q. Ma, R. Venugopalan, K. Watanabe, H.-F. Zhang, $\psi(2 S)$ versus J/ $\psi$ suppression in proton-nucleus collisions from factorization violating soft color exchanges, Phys. Rev. C97 (1) (2018) 014909.

[22] M. Laine, O. Philipsen, P. Romatschke, M. Tassler, Real-time static potential in hot QCD, JHEP 03 (2007) 054.

[23] P. Petreczky, A. Rothkopf and J. Weber, Realistic in-medium heavy-quark potential from high statistics lattice QCD simulations, arXiv:1810.02230 [hep-lat].

[24] J. P. Blaizot and M. A. Escobedo, Approach to equilibrium of a quarkonium in a quark-gluon plasma, Phys. Rev. D 98, no. 7, 074007 (2018) doi:10.1103/PhysRevD.98.074007

[25] B. Krouppa, A. Rothkopf, M. Strickland, Bottomonium suppression using a lattice QCD vetted potential, Phys. Rev. D97 (1) (2018) 016017.

[26] B. Krouppa, A. Rothkopf, M. Strickland, Bottomonium suppression at RHIC and LHC, in: 27th International Conference on Ultrarelativistic Nucleus-Nucleus Collisions (Quark Matter 2018) Venice, Italy, May 14-19, 2018, 2018. 